\title{
AUTONOMÍA EN EL APRENDIZAJE ¿ASPIRACIÓN EDUCATIVA O REALIDAD? EL IMPACTO DEL PROCESO FORMATIVO ESCOLAR
}

\author{
AUTONOMY IN LEARNING, EDUCATIONAL ASPIRATION OR \\ REALITY? THE IMPACT OF THE SCHOOL TRAINING PROCESS
}

\section{Roxana Lilian Arreola Rico* Concepción Hernández**}

Rec.: 14-03-2021. Acept.: 22-05-2021. Publ.: 30-06-2021

DOI: http://doi.org/10.29035/ucmaule.60.51

\section{RESUMEN}

El presente artículo analiza los conceptos y características de la autonomía, aprendizaje autónomo, las formas de cómo pueden favorecerse en el alumnado, las condiciones que benefician u obstaculizan el desarrollo de este tipo de aprendizaje, así como la función y la aportación que tiene en el proceso de formación; finalmente, se presenta una propuesta de intervención a fin de reorientar las prácticas docentes. El objetivo fue indagar sobre los factores de los procesos formativos formales y no formales que inciden en el desarrollo autónomo, promotores del aprendizaje independiente y de las creencias de autoeficacia, así como los significados y prácticas que tienen los docentes-estudiantes en torno a este tipo de aprendizaje. El trabajo es producto de una investigación de enfoque metodológico mixto que aborda el nivel de posgrado con profesores de educación básica, alumnos de la Universidad Pedagógica Nacional (México). Entre los hallazgos identificamos una clara relación entre el tipo de educación, la disciplina de crianza y el desarrollo de una autonomía académica, por lo que presumimos que la aspiración educativa de formar sujetos autónomos no es responsabilidad exclusiva de la escuela, sin embargo, la labor docente tiene una gran relevancia en la promoción de procesos de desarrollo autónomo.

Palabras clave: autonomía, aprendizaje autónomo, autorregulación, metacognición

\footnotetext{
Universidad Pedagógica Nacional, Unidad Sur, México, roxarreola@yahoo.com.mx https://orcid.org/0000-0002-3779-1788

** Universidad Pedagógica Nacional, Unidad Sur, México, concehdz@yahoo.es
} 


\section{ABSTRACT}

This article analyzes the concepts and characteristics of autonomy, autonomous learning and self-regulated learning, the ways in which they can be favored in students, the conditions that benefit or hinder the development of this type of learning, as well as the function and contribution they have in the learning process; finally, an intervention proposal is presented in order to reorient teaching practices. The objective was to investigate the factors of formal and non-formal learning processes that affect autonomous development, promoters of independent learning and self-efficacy beliefs, as well as the meanings and practices that teacher-students have around this type of learning. This work is the product of a mixed methodological approach research that addresses the postgraduate level students of the Universidad Pedagógica Nacional (Mexico) who are teachers of basic education. Among the findings we identify a clear relationship between the type of education, the parenting discipline and the development of an academic autonomy, so we presume that the educational aspiration to train autonomous subjects is not the exclusive responsibility of the school, however, the teaching practice has great relevance in promoting autonomous development processes.

Key words: autonomy, autonomous learning, self-regulation, metacognition

\section{INTRODUCCIÓN}

La autonomía es una cualidad que implica todo un proceso del individuo, dicho proceso inicia en la infancia y se espera que se concrete al término de la adolescencia, sin embargo, frecuentemente esto no se logra en el transcurso de toda la vida. Se trata de un proceso de diferenciación de carácter emocional y subjetivo que conforma la identidad, misma que implica la aceptación de sí mismo y la autonomía en la toma de decisiones, entre ellas: lograr el establecimiento de la elección vocacional y ocupacional, la capacidad para relacionarse en forma progresiva más real con los demás, la capacidad de establecer la relación con la pareja, así como conducirse por la vida a través de un conjunto de convicciones personales que guían y norman su comportamiento. Para Catherina de Luca (2009), la autonomía se entiende como la libertad que tiene una persona para decidir por sí mismo, expresar sus ideas, sentimientos y emociones sin restricciones. 
Desde una perspectiva filosófica y psicológica, la autonomía se entiende como la capacidad de los individuos para actuar como miembros responsables de una sociedad, mientras que, en el ámbito político, este término implica libertad de controles externos a la persona. Al respecto, Holec (1981) enfatiza en la autonomía concebida como un eje principal para alcanzar la libertad como individuos y el desarrollo de habilidades que nos permitan tener una posición más responsable e informada en el manejo de múltiples asuntos que conciernen a la sociedad en que vivimos (Viáfara y Ariza, 2008). Para nosotros, la idea de autonomía implica la capacidad que tienen los individuos para identificar sus potencialidades y limitaciones. Lo que implica asumir con responsabilidad su proceso de aprendizaje.

Para Díaz, Quiroga y Buadas (2014), la autonomía es un proceso de autorregulación. Es decir, la capacidad de las personas para manejar su propia conducta. Desde una perspectiva pedagógica, se la considera el paso previo de un aprendizaje activo y autónomo.

\section{AUTONOMÍA Y APRENDIZAJE}

Little (1991) y Low (1996) coinciden en su apreciación de la autonomía como la capacidad que tienen los estudiantes para tomar distancia, embarcarse en una reflexión crítica, llevar a cabo procesos de toma de decisiones y actuar independientemente. Así, este concepto es nuclear en la concepción de un estudiante que tiene la capacidad de controlar su propio aprendizaje, lo que implica la proposición de objetivos, la elección de materiales, tareas, formas de explotarlos y de plantear criterios definidos para autoevaluarse.

De acuerdo con Medina (s.f.), la autonomía implica tomar decisiones sobre la realidad y asumir el riesgo derivado de las mismas, tanto individual como socialmente. La autonomía ha representado el modo personal de conducirse en diversas situaciones, asumiendo el proceso de realización personal y eligiendo las decisiones personales más adecuadas para resolver los problemas que le atañen. La autonomía se genera en síntesis sucesivas de opciones que se afianzan cuando tales elecciones se integran en un sistema propio de realización. Pero la autonomía no solamente se construye con referencia a la independencia de elecciones responsables, sino también por la elaboración de criterios cada vez más fundamentados. 
El medio escolar ocupa un lugar importante en el desarrollo emocional y cognoscitivo porque proporciona un espacio para el aprendizaje formal y el ejercicio de la autonomía del alumno, al tiempo que las funciones psicológicas de percepción, pensamiento y juicio adquieren una nueva dimensión por medio de la abstracción (Bautista, 2005). Los maestros ocupan un lugar de extrema importancia en la formación de los adolescentes por los efectos que su actuación ejerce, en tanto que representan figuras sustitutas de los padres y como figuras disponibles para que los alumnos depositen las cargas afectivas provenientes de su infancia y desarrollen sus identificaciones.

\section{APRENDIZAJE AUTÓNOMO}

Las concepciones sobre cómo se adquieren conocimientos y cómo se aprende han cambiado a lo largo de la historia. En la antigüedad se pensaba que se lograba desde la hetero-estructuración del aprendizaje, donde el maestro elegía y regía la instrucción para sus estudiantes. Posteriormente, en la época moderna se consideró que el conocimiento se adquiere desde la autoestructuración del aprendizaje como una competencia esencial del sujeto. En cuanto a la época contemporánea, se establece una propuesta en la que el conocimiento se adquiere a través de la inter-estructuración del aprendizaje, por eso es tarea del que aprende, del que enseña a aprender y del grupo al que pertenece. Es decir, actualmente se asume que el aprendizaje es resultado de la interacción entre sujetos que, a su vez, se sitúan en un contexto sociocultural y natural determinado. Por lo tanto, no se puede hablar de independencia absoluta, solamente del desarrollo de estrategias, competencias de autorregulación y autonomía.

El aprendizaje autónomo, entonces, se refiere al grado de intervención del estudiante en el establecimiento de sus objetivos, procedimientos, recursos, evaluación y momentos de aprendizaje. Esto genera un rol activo mediante el cual el estudiante puede y debe aportar sus conocimientos y experiencias previas, mediante los cuales se pretende revitalizar y otorgar significado al aprendizaje. Así, el aprendizaje autónomo constituye un proceso intelectual mediante el cual el sujeto ejecuta estrategias cognitivas y metacognitivas, secuenciales, objetivas, procedimentales y formalizadas para obtener conocimientos estratégicos. Este proceso se rige por principios de acción: un interés manifiesto en razones que motiven la actuación deliberada, el reconocimiento de experiencias de aprendizaje previas, el establecimiento de nuevas relaciones entre aprendizaje-trabajo-vida cotidiana, entre teoría, práctica e identificación de la motivación intrínseca y el desarrollo del potencial personal de la autorregulación. 
El aprendizaje autónomo es un proceso donde el estudiante autorregula su aprendizaje y toma conciencia de sus propios procesos cognitivos y socioafectivos. La autorregulación implica tener conciencia del propio pensamiento. Es el conocimiento acerca de cómo se aprende. Para ello el sujeto debe observar, vigilar y controlar sus comportamientos para obtener un aprendizaje efectivo (Crispín, 2011). En consecuencia, con la autonomía académica se espera que el estudiante sea capaz de fijar compromisos y responsabilizarse de su aprendizaje, de manera que su autoevaluación le permita retomar los contenidos de aprendizaje cuantas veces sea necesario de acuerdo con las necesidades que se le presenten. De igual forma, se espera que pueda beneficiarse de las oportunidades que le ofrece la institución, así como la consulta en las bibliotecas virtuales y los materiales electrónicos.

Según Reyes (2017) el aprendizaje autorregulado más allá de ser un rasgo personal que los estudiantes pueden poseer, es la articulación de procesos específicos que involucran desde el planteamiento de objetivos, la posterior búsqueda y selección de estrategias, el monitoreo de su propio proceso, el ajuste de factores físicos y sociales para cautelar el logro de los objetivos, lo que implica el control efectivo del tiempo, la autoevaluación de los métodos usados y la reflexión acerca de los efectos, hasta la anticipación de futuros posibles métodos.

A decir de Arriola (2001, citado en Crispín, 201 1, p. 50) para favorecer el desarrollo de los procesos de autorregulación, los alumnos deben aprender a planificar, monitorear y valorar de manera consciente las actitudes y limitaciones con respecto a las demandas cognoscitivas que implica una tarea específica. Por lo que es necesario:

1. Planear: metas y actividades para el cumplimiento de la tarea.

2. Monitorear: comprensión del proceso de realización de la tarea y la reorientación de estrategias.

3. Valorar: comprensión de la eficacia con la que se desarrolla la actividad verificando la correspondencia entre el esfuerzo invertido y los resultados.

En adhesión a lo anterior, Bautista (2005) señala que lograr un aprendizaje autónomo implica que el alumno sea capaz de tomar decisiones en el aprendizaje, asumir compromiso en sus tareas escolares, desarrollar un nivel de tolerancia a la frustración y una necesidad de logro que le motive a plantearse retos en el aprendizaje, así como también a fijarse horarios de estudio accesibles y límites cuando sea necesario. En tanto que la labor del maestro enfocada al aprendizaje del alumno, en ambientes 
presenciales o virtuales, deberá ofrecer apoyo para el mejor aprovechamiento de sus funciones cognoscitivas que se traducirán en capacidad de juicio y de autocrítica para el logro de su identidad.

Luna y Sánchez (2005) establecen cuatro perfiles que describen las características referentes a la autonomía en los estudiantes participantes: 1) estudiantes que buscan alcanzar sus objetivos y, para lograrlo, actúan con un alto grado de independencia en las decisiones y recursos dentro del contexto educativo; 2) estudiantes que adoptan actitudes para aprender por sí mismos, pero cuyo compromiso no se revela dentro de su contexto académico, sino fuera de este; 3 ) estudiantes dependientes de las dinámicas de trabajo que plantean su grupo y el maestro; 4) estudiantes que, por su iniciativa y capacidad reflexiva, muestran niveles iniciales de autonomía para su aprendizaje.

La habilidad para tomar decisiones debe fomentarse desde edades tempranas a través de pequeñas elecciones tales como: qué ropa desea ponerse o a qué quiere jugar, porque cuantas más opciones de elección se presenten el niño adquiere mayor autonomía y tiene mayores posibilidades de llegar a valerse por sí mismo. Es decir, desde los primeros años los padres pueden ir abonando al desarrollo de la autonomía personal, la cual será una primicia para el progreso del aprendizaje autónomo.

El tiempo que se invierte como familia es, en algunos casos, el elemento que determina la calidad autónoma del niño. Se complementa con otras interconexiones como la influencia de los medios de comunicación, la escuela u otras relaciones con miembros que reflejan dependencia en su quehacer, recordando que el ejemplo es una vía por la cual el niño tiende a adquirir sus propios quehaceres, formas de pensar y sentir, por lo que siempre se debe tener un canal abierto a la comunicación. En algunos casos, el tiempo que se pasa con los niños en el salón ha llegado a superar el tiempo que los infantes pasan con sus propios padres, por lo que la escuela puede convertirse en su motor formativo. La escuela como institución y la propia familia como núcleo social fungen como sistemas formativos, por ello deben actuar de forma coordinada y sus acciones son muestra del tipo de orientación que se genera, ya sea favoreciendo el desarrollo autónomo o no.

\section{APRENDER A APRENDER}

Para Cabrera (2009), aprender a aprender constituye la expresión de la autonomía en el aprendizaje del estudiante. Se sintetiza en la idea de ser una guía para dirigir, controlar y evaluar de forma autorregulada los procesos de adquisición de conoci- 
mientos a través del empleo de estrategias de aprendizaje con la finalidad de responder a las exigencias de la práctica formativa.

La autonomía en el aprendizaje significa que el sujeto es capaz de captar las exigencias de las tareas de aprendizaje, movilizar una serie de conocimientos, habilidades y hábitos integrados en torno a una dirección específica de aprendizaje, utilizándolos intencionalmente. Una dirección para lograrlo puede ser mediante estrategias para la autorregulación:

- Estrategias de autoevaluación del estudiante del nivel de logro mediante revisión continua de los avances, dificultades y éxitos en la tarea según la meta de aprendizaje, así como la generación de alternativas de solución y previsión de la efectividad de las estrategias utilizadas (evaluación de las tareas o actividades realizadas y de las estrategias empleadas).

- Estrategias para la motivación profesional a través de acciones que refuercen la autoconfianza, la capacidad para el diagnóstico de fortalezas, debilidades y posibilidades para superar dificultades en el proceso de aprendizaje, que orienten hacia la proyección futura en la profesión. 
Boekaerts propone un modelo de aprendizaje autorregulado. A continuación, se presenta dicho modelo adaptado por Sanz de Acedo (1998):

Figura 1.

Estrategias
Metacognitivas
- Representación mental de las
metas
- Diseño de un plan de acción
- Monitorear el proceso
- Evaluar los resultados

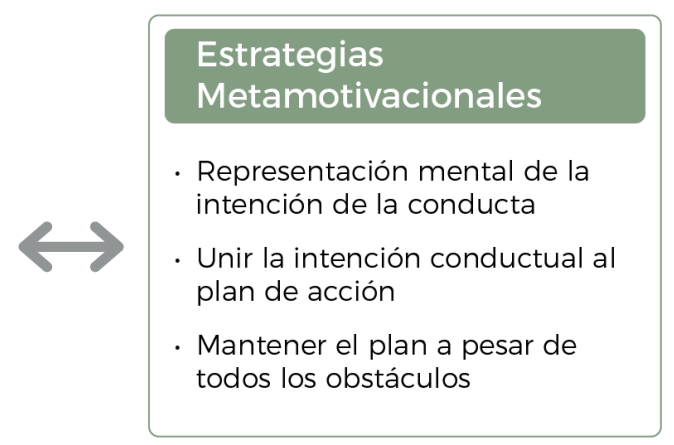

\section{Estrategias \\ Cognitivas}

- Atención selectiva

- Memorización

- Elaboración

- Organización

- Aplicar principios y reglas
Dominio de

Contenidos

- Conocimiento declarativo y procedimental generales y específicos.

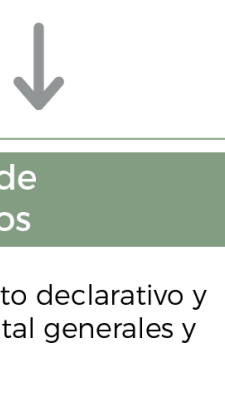

$$
\text { (n) }
$$

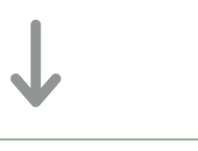

\section{Metamotivacionales}

Representación mental de la

Mantener el plan a pesar de todos los obstáculos

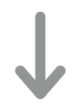

Estrategias

Motivacionales

- Generar deseos para aprender

- Controlar emociones

- Atribuciones internas o externas

- Evitar esfuerzo

- Pedir ayuda

\section{Creencias}

Motivacionales

- Creencias, actitudes y valores relacionados con las tareas de una materia

- Autoeficacia, es decir, confianza en las propias capacidades

Fuente: Crispín, M. (2011) 
En este punto recuperamos el concepto de autoeficacia, que se encuentra íntimamente relacionado con aspectos socioafectivos y emocionales en tanto este constructo se refiere a los juicios personales que se tienen de sí mismo con respecto a la capacidad de logro. Un estudiante puede poseer un concepto de autoeficacia positivo cuando se considera capaz de realizar eficientemente las tareas académicas, mientras que la autoeficacia negativa implica que el estudiante no se siente capaz de realizar tareas académicas y tiene la creencia de que está destinado al fracaso.

Bandura (1999) definió la autoeficacia percibida como los juicios personales que el sujeto tiene sobre sus propias capacidades para organizar o ejecutar cursos de acción con tal de lograr ciertas metas. Los criterios de la autoeficacia están referidos al autofuncionamiento futuro y son evaluadas antes de que los estudiantes realicen ciertas actividades relevantes. Así, la autoeficacia se refiere a las creencias de los individuos acerca de sus capacidades de aprender o realizar acciones en niveles designados como facilidad de aprendizaje o dificultad de la tarea. Este autor postulaba que las creencias de autoeficacia influyen sobre el nivel de esfuerzo, persistencia y selección de actividades. Los estudiantes con un alto sentido de eficiencia para completar una tarea educativa participarán con mayor disposición, se esforzarán más y persistirán durante más tiempo que aquellos que dudan de sus capacidades ante las dificultades. La autoeficacia percibida influye sobre el aprendizaje de los estudiantes a través de mecanismos cognitivos y motivacionales.

En términos ideales, maestros y padres deberán propiciar oportunidades para que el alumno desarrolle su autonomía en el contexto escolar, acompañando al estudiante en su aprendizaje, apoyándolo cuando sea necesario, pero también fijándole límites cuando se requiera. Sin embargo, esto no siempre sucede así, por el contrario, es frecuente que estos actores puedan convertirse en un obstáculo para el desarrollo de la autonomía y de las creencias de autoeficacia académicas.

Según Rayón, De las Heras y Muñoz (2011), el éxito en nuestra sociedad reside en que los sujetos sean capaces de aprender a lo largo de toda la vida, lo que les exige saber organizar y gestionar su propio aprendizaje. Esto requiere que los docentes seamos capaces de diseñar situaciones de aprendizaje en las cuales el alumnado debe controlar su propio proceso de aprendizaje, reflexionar y tomar conciencia de sus avances, dificultades y solicitar ayuda cuando la necesite, así como poder constatar cuándo está construyendo e integrando conocimiento. 


\section{PROBLEMÁTICA}

El desarrollo autónomo es una de las grandes aspiraciones de los procesos formativos, particularmente de la escuela. Sin embargo, al parecer también podría identificarse como un gran fracaso, pues cotidianamente es posible observar que las personas muestran comportamientos poco propositivos que evidencien iniciativa, libertad de pensamiento, acción con límites, elección y toma de decisiones argumentadas. Por el contrario, en los ambientes sociales, laborales y académicos suelen observarse prácticas orientadas al seguimiento de instrucciones, actitudes pasivas, poco propositivas, con tendencia a hacer lo que se espera de ellos en búsqueda de aprobación y a regirse por factores externos. La sociedad es la que apreciará de forma tangible el desarrollo crítico fundamentado en el aprendizaje autónomo, el cual, a su vez, se convierte en una herramienta para el aprendizaje permanente que ayude a responder a los retos actuales y el devenir de la vida.

Específicamente en el ámbito escolar, con frecuencia nos enfrentamos a estudiantes con escasa curiosidad e iniciativa, actitud pasiva e inerte, esperando respuestas por parte del docente, dependientes de compañeros o del propio profesor, inseguridad en el desempeño académico y con creencias o juicios de escasa capacidad de logro, situación que no se restringe a un nivel educativo específico ya que incluso permanece en niveles de educación superior como licenciatura y posgrado. Lo preocupante es que esta falta de autonomía académica no se limita al espacio escolar, sino que trasgrede muros y se aprecia en el comportamiento social cotidiano de los sujetos.

Al respecto, cabe preguntarnos ¿qué factores inciden en la conformación de los sujetos? ¿Cómo impactan los procesos formativos en el desarrollo de la autonomía académica y en las creencias de autoeficacia? ¿Cómo puede contribuir la escuela al desarrollo de la autonomía académica y las creencias de autoeficacia? ¿La escuela es la única responsable como agente formativo? Una vez que se respondan estas interrogantes, debemos trascender la perspectiva de investigación por el placer de conocer o explorar y avanzar hacia la solución de los problemas educativos; en este sentido, habremos de cuestionarnos sobre qué hemos hecho o qué hemos dejado de hacer para tener este perfil de estudiantes, ciudadanos y seres humanos y, a partir de esta reflexión, generar propuestas de intervención.

\section{OBJETIVO}

La investigación tuvo como objetivo identificar los factores o condiciones de vida en procesos formativos formales (escolares) y no formales (familiares) asociados con el 
desarrollo autónomo, como promotores de la autonomía académica y las creencias de autoeficacia en los docentes-estudiantes.

\section{METODOLOGIAA}

La investigación se fundamenta en propuestas de metodología mixta en tanto se recurre al análisis cuantitativo de datos, así como al análisis de corte cualitativo que otorgan importancia al aspecto subjetivo en la manera como se construye e interpreta la realidad social. Así, la información obtenida con cada uno de los instrumentos es procesada tanto cuantitativa como cualitativamente.

Recuperamos elementos que aporta el enfoque biográfico, de esta forma, hablamos de una investigación de carácter descriptivo, donde el relato del informante adquiere gran importancia.

Los relatos de vida forman parte del campo de la investigación cualitativa, cuyo paradigma fenomenológico sostiene que la realidad es construida socialmente mediante definiciones individuales o colectivas de una determinada situación (Taylor \& Bogdan, 1984). Es decir, se interesa por el entendimiento del fenómeno social desde la visión del actor. De ahí que los datos obtenidos al utilizar la metodología cualitativa consten de ricas descripciones verbales sobre los asuntos estudiados (Kavale, 1996 citado en Cordero, 2012). De acuerdo con Denzin (1978) el life story (relato de vida) designa la historia de una vida tal como la cuenta la persona que la ha vivido. La tarea del investigador es hacer visible o evidente las formas culturales y sociales presentes implícitamente en los relatos, situando la interpretación en un contexto que lo dote de sentido.

En el enfoque biográfico-narrativo se otorga mayor relevancia a la dimensión discursiva de la individualidad, a los modos como los humanos vivencian y dan significado al mundo de la vida mediante el lenguaje (Bolívar, 2014). En el caso concreto de esta investigación, se pretende que los docentes-estudiantes se expresen como autores de sus relatos de vivencias y prácticas cotidianas para, así, obtener elementos de significación, operatividad y autorreflexión que permitan identificar tanto problemáticas como avances en cuanto a la formación de los propios docentes-estudiantes.

Goodson et al. (2010) plantean que los relatos de vida no solamente reflejan aspectos de lo que las personas han aprendido en sus experiencias de vida, sino que, además, constituyen en sí mismos un proceso de aprendizaje. Es decir, el acto de narrar es un aprendizaje en acción en tanto es una práctica reflexiva de sus experiencias, como proceso estructurador de la construcción narrativa, organiza incidentes y acon- 
tecimientos heterogéneos de la vida en un orden (secuencial o temático) particular. Esta trama es lo que hace que el relato de vida sea una historia, singular y completa (Ricoeur, 2006).

\section{CONTEXTO DE INVESTIGACIÓN}

La experiencia se desarrolló al sur de la Ciudad de México en la Unidad 097 que forma parte de la Universidad Pedagógica Nacional, donde se imparte la Licenciatura en Educación y la Maestría en Educación Básica. Ambos programas se concentran en la formación profesional de docentes de educación básica en servicio, lo que genera un perfil particular que nos ha permitido identificarlos como docentes-estudiantes ya que desempeñan ambos roles de manera simultánea. En este sentido, el objetivo principal de estos programas educativos se orienta a la profesionalización de la práctica docente.

\section{ACTORES}

Como se ha referido, los actores que formaron parte de esta investigación actualmente cursan la Maestría en Educación Básica en la Universidad Pedagógica Nacional. La peculiaridad de estos alumnos es que de manera paralela se desempeñan como docentes en diferentes niveles educativos: preescolar, primaria o secundaria. De esta forma, el término más adecuado para referirnos a ellos es docentes-estudiantes.

\section{ESTRATEGIAS DE INDAGACIÓN}

Para la recopilación de información se utilizaron tres instrumentos que ofrecen distintos tipos de información que, en su conjunto, permiten una comprensión sustancial de la práctica cotidiana de los actores y que permite recuperar la significación de autonomía que le es conferida a posteriori por el sujeto:

a) Relato biográfico de los docentes-estudiantes. Se trata de un documento que recoge la narración de una experiencia vivida por una persona y expresada con sus propias palabras. Para la elaboración del relato se entregó previamente al informante un guion de preguntas o temas que se consideran esenciales para la investigación y que sirvieron como guía para el desarrollo del relato.

b) Cuestionario de estrategias de trabajo autónomo (CETA)

c) Lista de cotejo de rasgos de autonomía académica para identificar a los docentes-estudiantes que los evidencian durante la observación de clases. 


\section{RESULTADOS}

\section{Relato autobiográfico}

El relato autobiográfico permite identificar el tipo de educación y disciplina de crianza, el tipo de educación escolar, las formas de relación que establecen con la familia, los compañeros de trabajo y los mecanismos empleados para la toma de decisiones, los sentimientos de seguridad e inseguridad, así como experiencias de éxito y fracaso. A partir de ahí es posible generar una aproximación hacia las posibles relaciones entre estos aspectos con el desarrollo moral heterónomo o autónomo personal y en el aprendizaje. Para ello se elaboró un conjunto de categorías de análisis para los tres campos de indagación: historia familiar, historia escolar y contexto actual. Las categorías diseñadas fueron: dinámica familiar; disciplina heterónoma/autónoma; toma de decisiones; relación/comunicación; y vivencia de éxito/fracaso.

Al respecto, se encontró que de los 19 docentes-estudiantes 14 (73,7\%) evidenciaron haber tenido una educación y dinámica familiar autoritaria y heterónoma ya que los padres eran quienes tomaban decisiones, imponían reglas, normas, castigos y no dieron libertad para que ellos tomaran sus propias decisiones o participaran en el establecimiento de la disciplina y la normativa familiar. Hay que destacar que de estos 14 sujetos solo 3 (21,4\%) de ellos mostraron rasgos de autonomía, curiosamente se trata de los sujetos más jóvenes en los cuales, sugerimos, el contexto globalizador y cultural actual podría haber tenido mayor influencia. De los otros 5 sujetos que evidenciaron rasgos de una educación más democrática y en libertad, 4 describen en su relato características que corresponden a un desarrollo autónomo. 
Gráfico 1. Relación tipo de educación y crianza con autonomía académica.

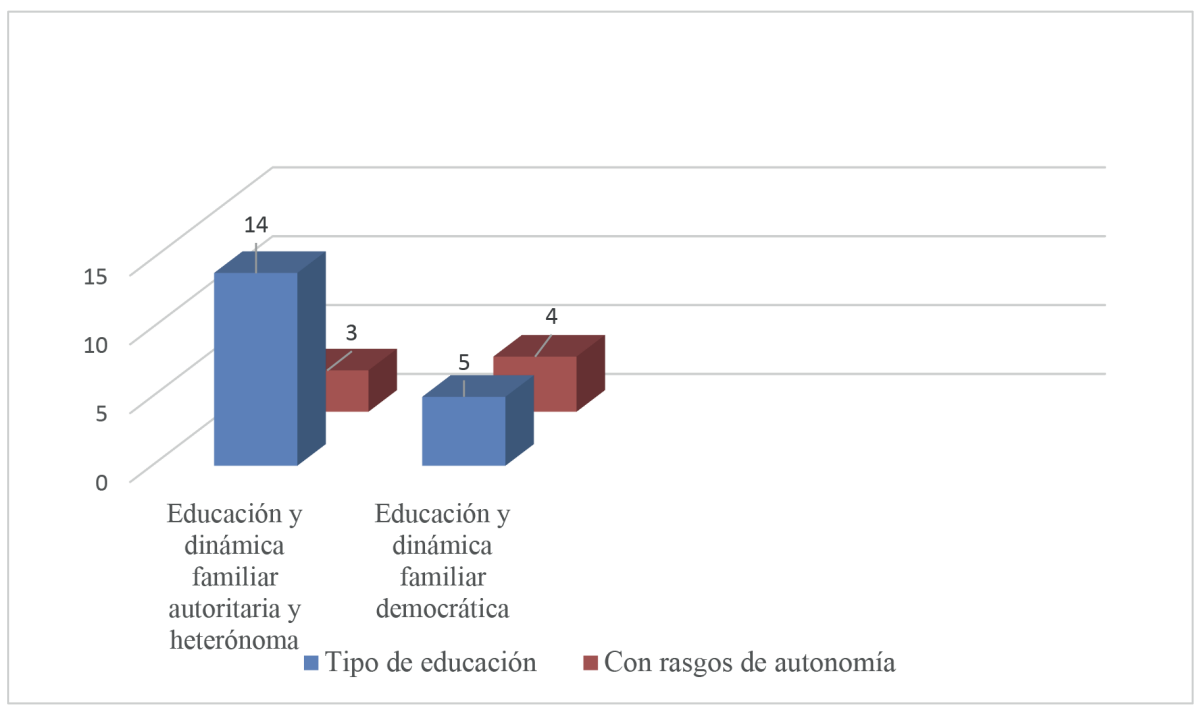

Fuente: elaboración propia.

Asimismo, se observa que las historias escolares asumen prioritariamente características de disciplina heterónoma, autoritaria, con altas limitaciones y escasa libertad de participación.

Con relación a la toma de decisiones, los sujetos que asumen características de educación y disciplina rígida y autoritaria utilizan como mecanismo para la toma de decisiones evitar castigos, sanciones o represalias. Están muy preocupados por lo que opinen los demás y los convencionalismos sociales, algunos incluso se sienten con poca capacidad de decisión, por lo tanto, dependen de los padres, cónyuges u otras personas para hacerlo. En contraste, los que adoptan características de una educación más democrática refieren tomar decisiones a partir del análisis y la reflexión de las consecuencias, de sus propios intereses y convicciones, toman iniciativa y distinguen cuándo deben seguir instrucciones por así convenir a sus intereses.

En cuanto a las experiencias de éxito y fracaso, se ha logrado identificar que los éxitos contribuyen fehacientemente a generar un sentimiento de seguridad, pero se discrimina claramente el ámbito al que corresponden: personal, escolar o laboral. Es decir, cuando se han tenido éxitos en el ámbito escolar se observa mayor seguridad en el rol de estudiante. Por otro lado, la prevalencia de fracasos provoca inseguridad. 


\section{Cuestionario de Estrategias de Trabajo Autónomo (CETA)}

Para analizar las estrategias de aprendizaje se tomó un instrumento elaborado por López-Aguado (2010) denominado Cuestionario de Estrategias de Trabajo Autónomo (CETA), el cual pretende explorar los comportamientos específicos de los alumnos en relación con las tareas de aprendizaje autónomo, que puede definirse como "modalidad de aprendizaje en la que el estudiante se responsabiliza de la organización de su trabajo y de la adquisición de las diferentes competencias según su propio ritmo. Implica asumir la responsabilidad y el control del proceso personal de aprendizaje, así como las decisiones sobre la planificación, realización y evaluación de la experiencia de aprendizaje" (Lobato, 2006, p. 191).

El cuestionario explora estrategias relacionadas con aspectos como planificación de tareas, búsqueda de información, comprensión y trabajo activo sobre la información, participación, relación con los compañeros, con el profesor, manejo de las nuevas tecnologías, entre otras.

El instrumento se divide en seis factores:

- Estrategias de ampliación. Describe estrategias relacionadas con la búsqueda, elaboración de materiales y actividades complementarias de ampliación del material propuesto por el profesor.

- Estrategias de colaboración. Describe una serie de estrategias relacionadas con la implicación del alumno en tareas grupales y de relación con otros compañeros.

- Estrategias de conceptualización. Reúne estrategias relacionadas con el trabajo intelectual sobre el contenido como elaboración de esquemas, resúmenes, mapas conceptuales, etc.

- Estrategias de planificación. Recoge aspectos relativos a la planificación de tiempos y programación de tareas.

- Estrategias de preparación de exámenes. Relacionadas con estrategias de estudio para exámenes, tales como selección de puntos importantes y actividades de repaso.

- Estrategias de participación. Describen el nivel de participación del alumno: asistencia a clases, aclaración de dudas, participación en el aula o tutorías. 
El conjunto de estas estrategias conlleva a considerar a la persona como agente activo de su propio aprendizaje, que aprende conforme a sus necesidades, intereses y potencialidades al ritmo que decide personalmente. El cuestionario ofrece una aproximación para la valoración del trabajo autónomo que constituye uno de los pilares de la formación por competencias.

De acuerdo con los factores antes señalados, se encontró que en cuanto a las "Estrategias de ampliación", los docentes-estudiantes, en promedio, manifestaron que en repetidas ocasiones realizan actividades para ampliar el material propuesto por el profesor, sin embargo, en un análisis más detallado se puede observar que de los 19 participantes 3 (16\%) pocas veces lo realizaron. Cabe señalar que esto no es evidente en las observaciones de clase ya que los estudiantes reducen su participación a las lecturas solicitadas por el docente y tampoco se nota el uso de alguna otra fuente para complementar sus participaciones.

En relación con el segundo factor, “Estrategias de colaboración”, se encontró que en promedio los sujetos pocas veces se implican en tareas grupales o colaboran con sus compañeros, esta situación es congruente con la observación de clase ya que el trabajo es generalmente individual, incluso cuando se les solicita alguna actividad en equipo ésta se reduce a la repartición de actividades por separado sin que exista un verdadero trabajo colaborativo.

En cuanto al tercer factor, "Estrategias de conceptualización”, mencionaron en promedio que solamente algunas veces realizan actividades de elaboración y tratamiento sobre el contenido a aprender. Esto es preocupante ya que refleja que los estudiantes se orientan hacia un aprendizaje memorístico, o bien, no se esfuerzan por hacer significativo su aprendizaje ni por darle un tratamiento a la información o a los aprendizajes.

Respecto a las “Estrategias de planificación", los participantes mencionaron que en repetidas ocasiones distribuyen tiempos, organizan actividades y tareas conforme a sus tiempos. Sin embargo, 8 de ellos (42\%) señalaron no hacerlo nunca o pocas veces, lo cual nos habla de un escaso compromiso hacia el aprendizaje y una baja capacidad de autonomía para organizarse.

El quinto factor, "Estrategias de preparación a exámenes", revela que en promedio los estudiantes realizan actividades de repaso y preparación para los exámenes. Por otro lado, con los resultados del cuarto y quinto factor se puede inferir que la preparación de exámenes responde más a la obtención de una calificación y la exigencia 
institucional que a su compromiso o autonomía hacia el aprendizaje, lo que implicaría un mayor peso de factores externos sobre intereses intrínsecos.

El sexto factor, “Estrategias de participación”, muestra que en promedio los participantes a veces tienen un nivel de participación bueno como alumnos, lo cual se hacen evidente mediante la asistencia a clases, aclaración de dudas, participación en el aula o en tutorías.

Por último, de acuerdo con el promedio de calificación que obtuvo cada uno de los participantes en los seis factores del cuestionario, se establecieron niveles de autonomía y de acuerdo con ellos se encontró que solo 2 estudiantes (10,5\%) demuestran un claro nivel de autonomía, 10 (52,6\%) presentan algunos rasgos de autonomía y 7 (36,8\%) evidenciaron no ser estudiantes autónomos.

\section{Gráfico 2. Estrategias de trabajo autónomo.}

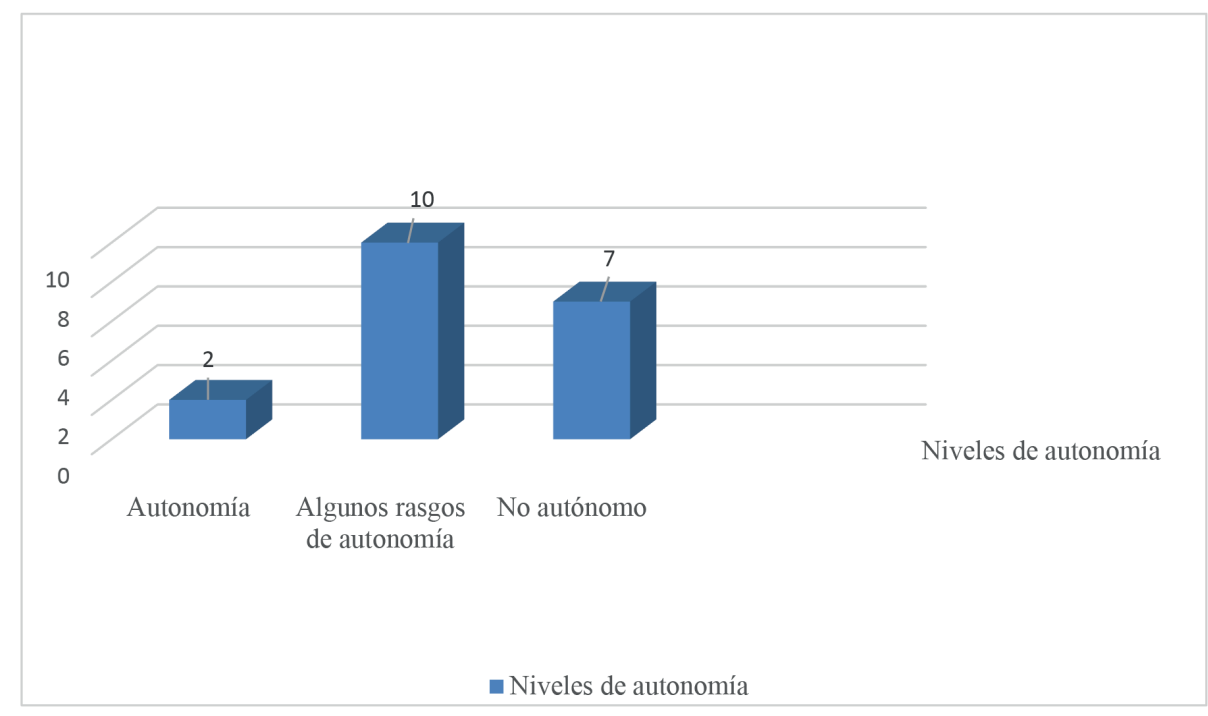

Fuente: elaboración propia

Relación Observación en clase e información obtenida de instrumentos de diagnóstico

La observación se llevó a cabo de manera directa y mediante el análisis de videograbaciones. Para el registro de la observación se elaboró una lista de cotejo en la que se definieron los criterios a observar como rasgos de autonomía, recuperando los planteamientos teóricos señalados al inicio de esta investigación. 
Al respecto, los resultados obtenidos en el cuestionario CETA empatan parcialmente con lo observado ya que, de los 19 participantes, 14 de ellos mostraron congruencia y consistencia entre su discurso y lo que se observó en clase. En cuanto a los 5 participantes restantes, se encontraron inconsistencias entre los observado en clase y lo consignado en sus cuestionarios CETA: 4 de ellos señalaron poseer características de autonomía, pero esto no se evidenció en el aula, por el contrario, 1 participante minimizó estas características a pesar de que su comportamiento en clase demostró importantes rasgos de autonomía.

En cuanto a la posible relación que puede existir entre el tipo de educación y disciplina de crianza con el desarrollo moral heterónomo o autónomo se realizó un análisis detallado sujeto por sujeto a fin de encontrar dicha relación. Por su parte, se puede decir que en la mayoría de los casos (14) existió una clara relación entre el tipo de educación y disciplina de crianza con la autonomía, es decir, los que manifestaron particularidades de tipo heterónoma son los que no evidencian rasgos de autonomía académica, y los que mostraron características de una educación y disciplina más democrática son quienes muestran autonomía académica en su rol de estudiantes. Únicamente en 5 casos no se encontró relación, en 4 de ellos a pesar de haber tenido una educación autoritaria, poco permisiva y heterónoma, los sujetos lograron desarrollar su autonomía académica y 1 que menciona haber tenido una educación democrática no demostró ser autónomo.

Al respecto es importante destacar que en el desarrollo de la autonomía influyen otros agentes y factores, si bien se puede observar que la familia y la escuela son fundamentales para dicho desarrollo, no podemos descartar que las experiencias de vida, los iguales, las características individuales, las figuras de autoridad o de apego ajenas a los padres y la escuela pueden ser aspectos que puedan contribuir u obstaculizar el desarrollo autónomo. Esta consideración podría explicar la porción minoritaria de casos en los que no se encontró relación entre el tipo de educación y la disciplina de crianza.

\section{DISCUSIÓN Y CONCLUSIONES}

En síntesis, identificamos una clara relación entre el tipo de educación, la disciplina de crianza adoptada en la familia y el desarrollo de una autonomía académica mostrada en los docentes-estudiantes del posgrado, ya que en la mayoría de los casos sí se encontró que la educación, la disciplina familiar y la escolar de corte más democrático favorecen el desarrollo de la autonomía. Por el contrario, una de corte autoritario e impositiva promueve un desarrollo heterónomo, pasivo y sumiso en el rol del estudiante. 
Por último, se puede identificar el papel tanto de los padres como del docente como agentes promotores de la formación y desarrollo de estrategias de aprendizaje autónomo. En este sentido, como señalan Díaz, Quiroga y Buadas (2014), la autonomía es el resultado de un proceso de desarrollo no tan sólo individual sino social también. Así, la vertiente humanística asume que la autonomía está relacionada a la cultura y a la dinámica social que permite que la persona incorpore valores y actitudes nuevas a su persona.

Por lo anterior, podemos presumir que la aspiración educativa de formar sujetos autónomos no es responsabilidad exclusiva de la escuela, sin embargo, sí podemos destacar que las prácticas educativas directivas, instructivas, de poca libertad y de sumisión, así como las prácticas de disciplina escolar centradas en el autoritarismo, la imposición y el castigo, que aún siguen prevaleciendo en las aulas, juegan un papel importante en la obstaculización del desarrollo de la autonomía, no solo de índole académica sino incluso personal.

Se puede concluir que la labor docente tiene una gran relevancia en la promoción de procesos de desarrollo autónomo, considerando que la promoción de la autonomía en el aprendizaje significa fomentar la capacidad de tomar decisiones, así como la independencia y la responsabilidad personal de los que están aprendiendo.

El tema de promover el aprendizaje autónomo desde las aulas ha cobrado cada vez mayor importancia, de acuerdo con Reyes (2017) durante los últimos años diversos estudios se han centrado en el aprendizaje autorregulado y han ofrecido importantes aportes al aprendizaje académico, entre ellos (Álvarez, 2009; Daura, 2010; Gaeta, Teruel y Orejudo, 2012; Lamas, 2008; Gargallo, Suarez y Ferrera, 2007; Zulma Lanz, 2006; Núñez et. al., 2006; Núñez et. al., 2008; Rinaudo, Chiecher y Donolo, 2003; Rosário et. al., 2012; Salmeron y Gutiérrez, 2012; Valle et. al., 2010).

Por otro lado, desde hace más de una década, Sierra (2005, p. 7) con el afán de darle prioridad al tema, mencionaba que "el aprendizaje autónomo tiene que ser una política curricular de la educación y no una posibilidad ofrecida por algunos docentes o tutores conscientes de esta necesidad y ventaja. En este sentido, el desarrollo de las habilidades del aprendizaje autónomo no debe dejarse al azar, sino que debe ser parte de la cultura institucional que se promueva desde las aulas, así como la implementación de acciones para fomentar el desarrollo de estas habilidades.

De ahí que consideramos necesario generar una propuesta para el trabajo docente, misma que se plantea a continuación. 


\section{PROPUESTA PARA LA INTERVENCIÓN DOCENTE}

Con esta propuesta se trata de superar la dinámica de la clase magisterial y centrar la atención en el aprendizaje y en la formación del estudiante, se requiere poner a disposición de los alumnos metodologías y estrategias formativas basadas en el aprendizaje autónomo y colaborativo, reconocer a los estudiantes como sujetos activos con capacidad para la reflexión, la producción creativa de conocimiento y la acción crítica.

Es evidente que las organizaciones escolares actualmente requieren profesionales capaces de detectar necesidades de formación, creativos en la identificación de problemas y en su resolución, con aptitudes para aprender de manera autónoma. Estas cuestiones son fundamentales para que sean capaces de construir, organizar y gestionar su aprendizaje a lo largo de la vida. La enseñanza que promueve el aprendizaje autónomo será aquella que se apoye en una concepción abierta e interactiva y sitúe a cada alumno, equipos de alumnos y clase como protagonistas de su aprendizaje.

Figura 2.

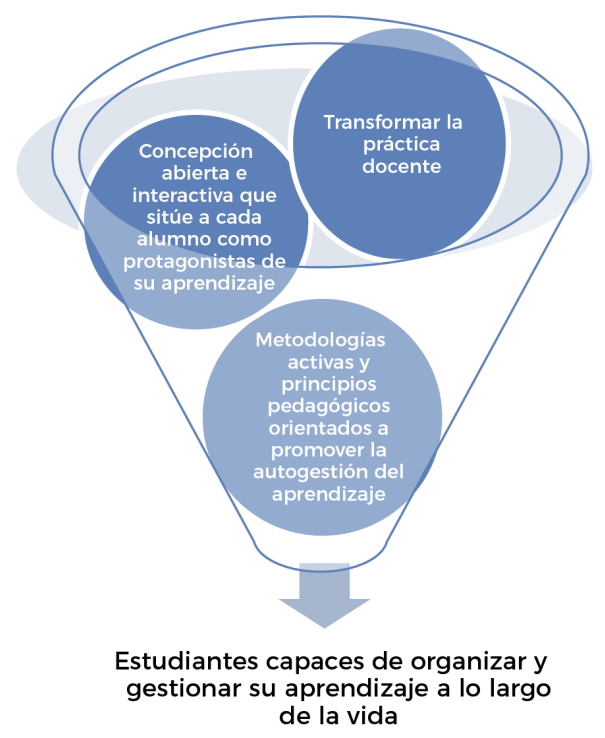

Fuente: elaboración propia. 
Para actuar y favorecer el aprendizaje autónomo se requiere tener en la docencia como principios pedagógicos los siguientes:

a) Ser estratégico generando condiciones para que el estudiante aprenda a aprender. Es decir, debe planear, conducir y adecuar las actividades al grupo y evaluar el logro de metas, no aspectos de disciplina o ajenos a dichas metas.

b) Establecer claramente y, de manera negociada, el objetivo de trabajo para que el alumno tome conciencia de la demanda y de lo que se espera de él.

c) Permitir que los estudiantes participen en la organización del trabajo académico, la toma de decisiones, sean propositivos, es decir, posibilitar el desarrollo de habilidades de autogestión de su aprendizaje.

d) Promover la participación en la organización y toma de decisiones de las actividades académicas, así como la proyección de las decisiones tomadas en diversos contextos: aula, sociedad, espacios abiertos y comunicación global, así como en diferentes esferas de su vida: académica, personal, familiar, social, laboral, etc.

e) Incorporar el uso de metodologías activas que promuevan la participación del estudiante en su proceso de aprendizaje.

f) Generar un clima social de autonomía y confianza, de potenciación relacional de los miembros del aula, promoviendo la acción colaborativa. El clima social posibilita que la interacción entre profesor y alumnos y de éstos entre sí se oriente en una u otra dirección formativa.

g) Promover el uso de técnicas de aprendizaje y metacognitivas que favorezcan la autorregulación de su proceso de aprendizaje, como señalan Valle et. al., 2007 (citado en Crispín, 2011) saber cómo, cuándo y por qué utilizarlas. Saber en qué circunstancias es más útil una estrategia sobre otra, modificarla según las demandas mediante la observación de la eficacia de las estrategias seleccionadas, cambiarlas o ajustarlas según las metas. Modelar las estrategias, hacer explícito cuándo y por qué usar esa estrategia y cuáles son los pasos que siguen.

h) Ayudar al estudiante a tomar conciencia de las estrategias que está utilizando, apoyarlo a reflexionar sobre sus propios procesos. Esto se puede hacer pidiendo al estudiante que explique los pasos que siguió para realizar determinada tarea. 
i) Problematizar el conocimiento mediante la lectura, el análisis de problemas o situaciones cotidianas y su posterior debate. Es importante iniciar con tareas que estén de acuerdo con el nivel de desarrollo cognitivo del estudiante, cercanas a su desarrollo próximo; promover el uso de conflictos cognitivos que no sean ni tan fáciles que no les impliquen un reto ni tan difíciles que no las puedan realizar.

j) Promover el gusto por aprender, la satisfacción y la motivación intrínseca, es decir, la satisfacción interna y no centrase sólo en las calificaciones. Explicitar la importancia de la automotivación para lograr las metas deseadas, así como la voluntad y la perseverancia para conseguirlas.

k) Procurar que el estudiante tome conciencia de todas las actividades que tiene que realizar para que pueda hacer cronogramas y horarios que le permitan organizar mejor su tiempo.

I) Procurar que el alumno se sienta seguro y capaz de realizar la tarea encargada. Para ello es necesario empezar con tareas que el alumno pueda realizar $y$, poco a poco, aumentar el grado de dificultad. Hay que recordar que las expectativas que tiene el profesor hacia los estudiantes suelen cumplirse. En este sentido, es importante motivar altas expectativas a los estudiantes para generar confianza en sus propias capacidades.

m) Relacionar la teoría y la práctica de modo que los alumnos puedan transitar fácilmente de los planteamientos o conceptos a realidades concretas, reales y viceversa, mediante metodologías que impliquen actuaciones interactivas.

n) Favorecer el desarrollo del pensamiento crítico.

o) Diseñar situaciones en las cuales los alumnos controlen su propio proceso de aprendizaje, reflexionen, tomen conciencia de sus avances, dificultades y soliciten ayuda cuando la necesite, así como poder constatar cuándo se está construyendo e integrando conocimiento. Es importante que los estudiantes se conozcan a sí mismos, que tengan un diálogo interno de modo que reconozcan sus fortalezas y aquellos aspectos que necesitan consolidar.

p) La retroalimentación que da el profesor es muy importante, idealmente debe ser en sentido positivo, señalar las fortalezas y los errores, pero en un tono que permita al alumno superarlos, reconociéndolos como una oportunidad de aprendizaje. 
q) Promover la autoevaluación para comprender las razones y los logros alcanzados en la construcción personal, académica y social de la realidad a través de la concienciación y reflexión sobre los aprendizajes realizados.

Finalmente, las sugerencias anteriores están orientadas a propiciar prácticas docentes en un ambiente de libertad, confianza y acompañamiento, a fin de que los estudiantes vayan incursionando en el desarrollo de procesos autónomos en torno al aprendizaje. Ello implica abandonar prácticas tradicionales que fomentan la sumisión, la pasividad, el seguimiento de instrucciones y la memorización mecanizada; por el contrario, se busca promover el espíritu crítico, reflexivo, innovador, activo y propositivo por parte de los alumnos que los acerquen a la autonomía del aprendizaje.

\section{REFERENCIAS BIBLIOGRÁFICAS}

Bandura, A. (1999). Auto-eficacia: cómo afrontamos los cambios de la sociedad actual. Bilbao: Desclée de Brouwer.

Bautista, Y. (2005). La autonomía del alumno en el aprendizaje. Reto del nuevo modelo educativo del IPN. Revista Innovación Educativa, 5(25), 41-54. Recuperado de https://www.redalyc.org/articulo.oa?id=179421454005

Bolívar, A. (2014) Las historias de vida del profesorado. Revista Mexicana de Investigación Educativa, 19(62), 911-734. Recuperado de https://www.redalyc.org/ pdf/140/14031461004.pdf

Cabrera, I. (2009). Autonomía en el aprendizaje: direcciones para el desarrollo en la formación profesional. Revista Electrónica Actualidades Investigativas en Educación, 9(2), 1-22. Recuperado de https://www.redalyc.org/ pdf/447/44713058006.pdf

Cordero, M. (2012). Historias de vida: Una mirada de investigación cualitativa. Revista Griot, 5(1), 50-67. Recuperado de https://www.uv.mx/psicologia/files/2017/12/ historias_de_vida_una_metodologia_de_investigacion_cualitativa.pdf

Crispín, M. (Coord.) (2011). Aprendizaje autónomo. Orientaciones para la docencia. México: Universidad Iberoamericana.

De Luca, C. (2009). Implicaciones de la Formación en la Autonomía del Estudiante Universitario. Electronic Journal of Research in Educational Psychology, 7, 901 922. Recuperado de https://www.redalyc.org/articulo.oa?id=293121945015 
Denzin, K. (1978). The research Act: A theoretical introduction to sociological methods. New York: Mc Graw Hill

Díaz, N., Quiroga, E. y Buadas, C. (2014). El desarrollo de la autonomía de los alumnos de inglés con fines específicos. En Cuadernos de la Facultad de Humanidades y Ciencias Sociales, 46, 179-194. Recuperado de https://www.redalyc.org/ articulo.oa?id=18542677010

Goodson, I., Biesta, G., Tedder, M. \& Adair, N. (2010). Narrative learning. Londres y Nueva York: Routledge.

Holec, H. (1981). Autonomy in Foreign Language Learning. Oxford: OUP.

Little, D. (1991). Learner Autonomy: Definitions, Issues and Problems. Dublin: Authentik Language Learning Resources Ltd.

Low, H. (1996). Encouraging learner independence. How a Colombia Journal for English Teachers, 1(1), 39-41. Recuperado de https://www.howjournalcolombia. org/index.php/how/article/view/287

Luna, M. y Sánchez, D. (2005). Profiles of autonomy in the field of foreign languages. Profile: issues in teachers' professional development, 6, 133-140. Recuperado de https://www.researchgate.net/publication/228953894_Profiles_of_Autonomy_in_the_Field_of_Foreign_Languages

Medina, A. (s.f.). Hacia un modelo de enseñanza que promueve el aprendizaje autónomo a distancia de las personas adultas. Recuperado de http://www.crefal. edu.mx/rieda/images/rieda-1995-3/articulo1.pdf

Rayón, L., De las Heras, A. y Y. Muñoz. (2011). La creación y gestión del conocimiento en la enseñanza superior: la autonomía, autorregulación y cooperación en el aprendizaje. Revista Iberoamericana de Educación Superior, 2(4), 103-122. Recuperado de http://www.scielo.org.mx/pdf/ries/v2n4/v2n4a6.pdf

Reyes, M. (2017). Desarrollo de la competencia de aprendizaje autónomo en estudiantes de Pedagogía en un modelo educativo basado en competencias. REXE Revista de Estudios y Experiencias en Educación, 16(32), 67-82. Recuperado de https://www.redalyc.org/pdf/2431/243153684004.pdf 
Ricoeur, P. (2006). La vida: un relato en busca de narrador. Ágora. Papeles de filosofía, 25(2), 9-22. Recuperado de https://minerva.usc.es/xmlui/bitstream/handle/10347/1316/Ricoeur.pdf?sequence=1

Sanz de Acedo, M.L. (1998). Inteligencia y personalidad en las interfases educativas. Bilbao: Desclée De Brouwer.

Sierra, J. (2005). Aprendizaje autónomo: eje articulador de la educación virtual. Revista Virtual Universidad Católica del Norte, Fundación Universitaria Católica del Norte Medellín, Colombia, 74, 1-7. Recuperado de https://revistavirtual.ucn. edu.co/index.php/RevistaUCN/article/view/261

Taylor, S. \& Bodgan, R. (1984). Introducción a los métodos cualitativos de investigación. Buenos Aires: Paidós.

Viáfara, J. y J. Ariza. (2008). Un modelo tutorial entre compañeros como apoyo al aprendizaje autónomo del inglés. Íkala, revista de lenguaje y cultura, 13(19), 173209. Recuperado de https://www.redalyc.org/articulo.oa?id=255020449009 